\title{
Digoxin use in patients with heart failure with reduced ejection fraction
}

\author{
Yaniel Castro-Torres* \\ Servicio de Cardiología, Hospital Universitario Celestino Hernández Robau. Santa Clara, Villa Clara, Cuba
}

Heart failure represents an important cause of morbidity and mortality worldwide. Despite recent advances in the treatment of this condition, majority of patients have a bad prognosis, low functional class and poor quality of life. Recent guidelines have approved new drugs for the management of these patients and many others are under investigation. ENTRESTO, a combination of sacubritil/valsartan and ivabradine reduce the risk of cardiovascular mortality in patients with heart failure with reduced ejection fraction and are recommended in patients who maintain symptomatic despite concomitant use of angiotensin-converting enzyme inhibitors, beta-blockers and aldosterone antagonists [1,2].

Digitalis was discovered and first implemented several centuries ago and has been widely used in clinical practice since the beginning of last century [3]. It has physiological properties that include improvement in cardiac output and peak oxygen consumption, and a decrease in pulmonary capillary wedge pressure and sympathetic overactivity [4].

Current guidelines approve the use of digoxin in patients with heart failure with reduced ejection fraction who remain symptomatic despite the use of angiotensin-converting enzyme inhibitors, betablockers and aldosterone antagonists, as first line therapy, and ENTRESTO, ivabradine and cardiac resynchronization therapy as second line therapy $[1,2]$.

So far, no studies have demonstrated that digoxin reduces mortality in patients with heart failure. DIG trial was the first randomized doubled-blind controlled study that evaluated the efficacy of digoxin in patients with heart failure with reduced ejection fraction. Main findings revealed that there was no difference in mortality between digoxin and placebo groups, but a reduction in hospitalization for heart failure worsening was found [5]. Several no randomized controlled trials and post hoc analysis of DIG trial have been published since that, and outcomes associated to digoxin use are essentially the same [4].

A recent observational trial evaluated digoxin use after hospital discharge in a cohort of 3062 matched patients with heart failure with reduced ejection fraction. They found that digoxin use was associated with a significant reduction in the risk of heart failure readmission at 30 days, 1 year and 6 years (HR: 0.74; 95\% CI: 0.59-0.93), (HR: 0.81; 95\% CI: $0.72-0.92$ ) and (HR: 0.90; 95\% CI: 0.81-0.99) respectively. However, digoxin administration was not associated with mortality rates after hospital discharge [6]. Another previous study conducted by Lam et al. [7] showed similar results. In this trial, patients had a mean age of 74 years, a mean left ventricular ejection fraction of $29 \%$ and all received beta-blockers at hospital discharge. After a long term follow-up, 30-day and 4-year all cause hospital readmission were less frequent in patients receiving digoxin compared with patients who did not receive digoxin at hospital discharge (HR: 0.51; 95\% CI: 0.31-0.83; $\mathrm{p}=0.007)$ and (HR; 0.72; 95\% CI: 0.57-0.92; $\mathrm{p}=0.008$ ) respectively.

These contemporary investigations support current recommendations and no additional evidence is advised to change actual medical practice. Both studies demonstrated the clinical effectiveness of digoxin in reducing the risk for readmission in hospitalized patients who are taking current guideline-based medical therapies. Past and present studies evaluating digoxin efficacy in clinical practice have not included current drugs and interventions which have demonstrated to reduce mortality. Thus, findings observed in present studies should stimulate further randomized trials that combine these therapies, especially in patients with low functional class, severely abnormal left ventricular ejection fraction and high comorbidity burden who are at higher risk for adverse clinical outcomes.

Although the level of evidence for digoxin use in patients with heart failure with reduced ejection fraction has declined over time, no randomized controlled trials have demonstrated that its administration is associated with an increased in mortality or severe side effects that motivate its definitive or progressive withdrawal in clinical practice. At the same time, a subgroup of high-risk patients such as older patients, diabetics and those with lower left ventricular ejection fraction have a higher prescription of digoxin, without differences in mortality compared with no digoxin treated patients [8].

Sex and ages differences, values of left ventricular ejection fraction, functional class, comorbidities and concomitant drug interactions associated to digoxin use are some controversial points which should be answered in further studies for improving clinical outcomes.

Based on its unique and well-demonstrated physiological properties and positive outcomes showed in contemporary studies, digoxin administration in patients with heart failure with reduced ejection fraction should be preserved, especially in combination with current guideline-based therapies in order to reduce hospitalizations and improve patients' quality of life.

\section{Conflict of interest}

None declared.

${ }^{*}$ Correspondence to: Luz Caballero 161 e/Hospital y Alejandro Oms. Santa Clara, Villa Clara, Cuba, Tel: 5353217694; E-mail: castrotorresy@gmail.com

Received: June 27, 2019; Accepted: July 18, 2019; Published: July 22, 2019 


\section{Funding}

None.

\section{References}

1. Yancy CW, Jessup M, Bozkurt B, Butler J, Casey DE Jr, et al. (2017) ACC/AHA/HFSA focused update of the $2013 \mathrm{ACCF} / \mathrm{AHA}$ guideline for the management of heart failure: A report of the American college of cardiology/American heart association task force on clinical practice guidelines and the heart failure society of America. Circulation 136: e137-e161. [Crossref]

2. Ponikowski P, Voors AA, Anker SD, Bueno H, Cleland JGF, et al. (2016) ESC Guidelines for the diagnosis and treatment of acute and chronic heart failure: The Task Force for the diagnosis and treatment of acute and chronic heart failure of the European society of cardiology (ESC). Developed with the special contribution of the heart failure association (HFA) of the ESC. Eur Heart J 37: 2129-2200. [Crossref]
3. Katholi RE, Castro-Torres Y, Ervin MR (2019) Pharmacological "game changers" for HFrEF patients. J Cardio Res 2: 15.

4. Albert CL, Kamdar F, Hanna M (2016) Contemporary controversies in digoxin use in systolic heart failure. Curr Heart Fail Rep 13: 197-206.

5. The digitalis investigation group (1997) The effect of digoxin on mortality and morbidity in patients with heart failure. N Engl J Med 336: 525-533.

6. Qamer SZ, MalikA, Bayoumi E, Lam PH, Singh S, et al. (2019) Digoxin use and outcomes in patients with heart failure with reduced ejection fraction. Am J Med. [Crossref]

7. Lam PH, Bhyan P, Arundel C, Dooley DJ, Sheriff HM, et al. (2018) Digoxin use and lower risk of 30-day all-cause readmission in older patients with heart failure and reduced ejection fraction receiving $\beta$-blockers. Clin Cardiol 41: 406-412. [Crossref]

8. Ziff OJ, Lane DA, Samra M, Griffith M, Kirchhof P, et al. (2015) Safety and efficacy of digoxin: systematic review and meta-analysis of observational and controlled trial data. $B M J$ 351: h4451. [Crossref]

Copyright: (C2019 Castro-Torres Y. This is an open-access article distributed under the terms of the Creative Commons Attribution License, which permits unrestricted use, distribution, and reproduction in any medium, provided the original author and source are credited. 